\title{
A visible light-activated direct-bonding material: An in vivo comparative study
}

\author{
K. D. O'Brien, BDS, FDS, MSc, D. Orth., ${ }^{*}$ M. J. F. Read, BDS, FDS, D. Orth., \\ R. J. Sandison, BDS, FDS, D. Orth., ${ }^{\star *}$ and C. T. Roberts, BSc, MSc, FSS* \\ Manchester, England, and Aberdeen, Scotland
}

\begin{abstract}
A clinical trial was carried out to evaluate and compare the clinical performance of a visible light-cured material with a chemically cured adhesive. This was used in combination with two types of bracket base. Fifty-two patients entered the trial and 542 bracket bases were placed. The incidence and site of bond failure were recorded. The overall failure rate for the light-cured material in combination with both types of bracket was $4.7 \%$ and $6 \%$ for the chemical-cured adhesive. There were no significant differences detected between the failure rates for both types of adhesive in combination with either bracket base, and no bracket base/adhesive combination proved superior $(p>0.05)$. When the data were examined in an overall manner, a significantly higher posterior tooth failure rate was detected for all adhesive/base combinations $(p<0.001)$. (AM J ORTHOD DENTOFAC ORTHOP 1989;95:348-51.)
\end{abstract}

$\mathrm{T}$ here have been many in vitro investigations into the use of directly bonded orthodontic brackets; these studies have attempted to identify the effects of the many variables that govern the strength of the bond..$^{1-4}$ However, optimum conditions for placement of brackets and moisture isolation exist in the in vitro situation and consequently in vitro testing provides only a guide to the selection of bracket/adhesive combinations. It has always been assumed that the clinical properties will be similar to the in vitro properties, ${ }^{5}$ which have been evaluated in the laboratory.

Several clinical investigations have been carried out into the failure rates of bonded brackets using chemically activated adhesives. Gorelick ${ }^{6}$ investigated 549 Concise $\uparrow /$ perforated base combinations and found a $4 \%$ failure rate for upper incisors and $7 \%$ for lower premolars. A similar investigation evaluating 705 attachments yielded a $10 \%$ failure rate for incisors and $29 \%$ for molars. ${ }^{7}$ A study that compared direct and indirect bonding techniques, ${ }^{8}$ using three different adhesives in combination with foil mesh and perforated bases, showed failure rates of $2 \%$ for the direct method and $13 \%$ for the indirect method from a total of 444 brackets.

The use of visible light-cured materials for orthodontic bonding was first described in $1980 .{ }^{9}$ The meth-

*Department of Orthodontics, University Dental Hospital, Manchester. **Department of Orthodontics, Royal Aberdeen Childrens' Hospital, Aberdeen.

$\dagger 3$ M Dental Products, St. Paul, Minn ods of use for the direct bonding technique have been described in which the adhesive material was cured by transillumination through the tooth structure. ${ }^{9,10}$ Lightcured materials also have been used in an indirect bonding technique in which the brackets are held in place before curing by means of a clear plastic mold. ${ }^{11}$ Laboratory investigations have suggested that they compare well with chemically cured adhesives ${ }^{12}$ and they may also have the advantage of leaving a reduced amount of residual debris after bracket removal.$^{13} \mathrm{~A}$ recent clinical investigation has been carried out into the use of a light-cured material in comparison with a chemically cured material; of the 1366 attachments that were placed, the failure rate for the chemically cured material was $16 \%$ and that for the light-cured material was $23 \% .^{14}$

\section{OBJECTIVE}

The objective of this study was to investigate and compare the clinical properties of a visible light-cured adhesive with a chemically cured material in combination with two different designs of bracket base.

\section{MATERIALS AND METHODS}

Two different designs of bracket base were used: a foil mesh* and a base that was indented and photoetched. $\dagger$ The foil mesh base has retention provided by a mesh of stainless steel wire $0.12 \mathrm{~mm}$ in diameter, woven to create openings of $0.20 \mathrm{~mm} .{ }^{2}$ The second

*Mini Mono, Forestadent, Milton Keynes, U.K.

†Micro-loc, GAC International, Inc., Central Islip, N.Y. 
base has retention provided by small indentations cut into the base, which is microscopically roughened by a photo-etching process.

One of the adhesives used was a chemically activated system* comprising a filled diacrylate resin and a low viscosity resin containing a tertiary amine activator, which is painted onto the etched enamel surface. Polymerization is initiated when the two components come into contact. 'The other adhesive was an experimental visible light-cured material $\dagger$ composed of a urethane dimethacrylate resin containing average sized filler particles $(13 \mu \mathrm{m}) ; 50 \%$ of the particles were less than $2 \mu \mathrm{m}$, resulting in a filler loading of $62.8 \%$ by volume. The catalyst system consisted of an $\alpha$ diketone and an amine; polymerization occurred on exposure to visible light of a wavelength of $470 \mathrm{~nm}$. The study used a single-blind controlled design, involving a withinpatient comparison of the two adhesives, with each patient randornly allocated two different bracket/adhesive combinations for each side of the mouth. A total of 542 brackets were bonded to 52 patients attending for routine fixed appliance treatment. The patients ranged in age from 13 to 29 years.

The bonding techniques were standardized and will be described in some detail; the following steps were taken. The teeth were isolated and polished with plain pumice and etched with $37 \%$ phosphoric acid for 60 seconds; they were then washed with copious amounts of water and dried with compressed air from an oil-free air supply. The bonding technique was different for the two materials.

Bonding technique for chemically cured material. The unfilled primer resin catalyst was applied with a brush to the retentive surface of the bracket bases and to the etched enamel surface. The filled resin was then loaded onto the bracket base and the based was pressed firmly onto the enamel surface, bringing the activator and initiator into contact, which caused polymerization to commence.

Bonding technique for light-cured material. The low viscosity resin was applied to the bracket base. This was polyrnerized by 10 seconds of exposure to a visible light source $\ddagger$ generating light with a wavelength of $470 \mathrm{~nm}$. The light source was shone directly onto the bracket to ensure that the resin cured completely in the indentations of the bracket base. This stage was carried out while the teeth were being etched so that there would be no delay in placement. The unfilled resin was also applied to the dried etched enamel surface;

*Lee unique, Lee Pharmaceuticals, South El Monte, Calif

†I.C.I. Dental, Macclesfield, U.K

\$Coe-lite, Cos Dental, Alsip, III. this was carried out immediately after the enamel surfaces were dried to prevent moisture contamination of the etched enamel. The filled resin phase was then loaded onto the bracket base with slight excess material and the bracket positioned firmly on the tooth. All the brackets were placed on the teeth before any curing took place; any excess adhesive material that had flowed from beneath the base was removed. When the operator was satisfied with the bracket positions, each of the brackets was cured singly, the adhesive being cured by exposure to the visible light by transillumination through the tooth structure. ${ }^{12}$ The light source was directed from the occlusal surfaces of each tooth for 30 seconds.

All brackets were placed by two experienced operators. A record of bracket failures and time of failure was kept for each patient. Data analysis was carried out by means of the Mantel-Haenszel chi-squared statistic. ${ }^{15}$

\section{RESULTS}

Fifty-two patients entered the study; the sample comprised 17 male and 35 female subjects. Of the total sample, 24 patients were fitted with mandibular and maxillary appliances, 22 were restricted to upper arch treatment, and six had mandibular appliances alone. Two hundred sixty brackets were attached with the light-cured material and 282 with the chemical-cured material, resulting in the placement of 542 attachments. All 52 patients were followed until the completion of treatment. Of these, $22(42.3 \%$ ) suffered bracket failure during treatment. The lengths of treatment ranged from 2 to 22 months, with mean and median treatment lengths of 12.4 and 14 months, respectively. The study was carried out throughout a 2-year period.

Malocclusions were present as follows: Class $I=14$ cases; Class II, Division $1=22$ cases; Class II, Division $2=8$ cases; and Class III $=7$ cases.

From a total of 542 brackets placed, 35 failed; all failures occurred within the first 12 months of treatment. Examination of the pattern of failure within the patients suggested an increased incidence of bracket failure in specific patients, with five patients suffering three failures (Table I). This within-patient effect is one of the justifications for the use of a within-patient comparison experimental design, and it is therefore necessary to take into account the within-patient effect in any statistical analysis. Because there was considerable variation in the length of treatment, it was decided to examine the outcome after a fixed period of time. Only those patients whose treatments extended to this time point would be included in the subsequent analysis. 
Table I. Incidence of bracket failure by patients

\begin{tabular}{c|c}
\hline No. of failures & No. of patients \\
\hline 0 & 30 \\
1 & 14 \\
2 & 2 \\
3 & 5 \\
\hline
\end{tabular}

Table II. Failure of individual bracket/base combinations

\begin{tabular}{lc|c|c}
\hline \multicolumn{1}{c|}{ Bracket/adhesive } & No. placed & Failed & Percentage \\
\hline Light cure/Mesh foil & 128 & 5 & $3.9 \%$ \\
Light cure/Micro-loc & 127 & 7 & $5.5 \%$ \\
Chemical cure/Mesh foil & 123 & 6 & $4.9 \%$ \\
Chemical curc/Micro-loc & 107 & 8 & $7.5 \%$ \\
Overall failure rates & & & \\
Light cure & 255 & 12 & $4.7 \%$ \\
Chemical cure & 230 & 14 & $6.0 \%$ \\
\hline
\end{tabular}

Thirty-seven percent (13) of the attachments failed within the first month and $82 \%$ (29) failed within the first 6 months. Accordingly, for clarity of analysis and comparability with other investigations, ${ }^{8}$ it was decided to compare the success or failure of each attachment at the 6-month time point. As a consequence eight patients who had completed treatment before 6 months were excluded from further data analysis.

The distribution of the various adhesive/base combinations and recorded bond failures after this 6-month time period are shown in Table II.

For the light-cured material in combination with both types of bracket base, the overall failure rate was $4.7 \%$; the failure rate for the chemically cured material was $6.0 \%$.

When the individual bracket base/adhesive combinations were examined, it was found that the lightcured material resulted in failure rates of $3.9 \%$ with the mesh foil base and $5.5 \%$ with the Micro-loc base; the chemically cured adhesive in combination with the foil mesh and Mico-loc bases yielded failure rates of $4.9 \%$ and $7.5 \%$, respectively.

Data analysis, with the Mantel-Haenszel chi-squared statistic, demonstrated the following:

1. No significant differences were detected between the failure rates for both types of adhesive in combination with both types of bracket $(p>0.1)$. No bracket/adhesive combination proved superior.

2. No bracket/adhesive combination resulted in
Table III. Failure rate for bracket position (bases and adhesives combined)

\begin{tabular}{l|c|c|c}
\hline Arch & Upper & Lower & Combined \\
\hline Anterior & $1.4 \%$ & $4.2 \%$ & $2.6 \%$ \\
Posterior & $16.1 \%$ & $12.56 \%$ & $11.8 \%$ \\
\hline
\end{tabular}

significantly less failures between the upper and lower arches $(p>0.1)$.

3. For both types of base in combination with both types of adhesive, highly significant differences were detected between anterior and posterior failure rates $(p<0.001)$, with the incidence of posterior failure being higher (Table III).

\section{DISCUSSION}

The overall failure rate recorded in this study when the chemical-cured material was used was similar to that obtained in previous investigations. ${ }^{6,7.9}$ When considering the light-cured material, the only published clinical trial produced a failure rate of $23 \%$; this is substantially higher than that achieved in the present study. It should also be noted that a similar high failure rate of $16 \%$ resulted with a chemically cured material. In the previous investigation, the light-cured material had a filler loading of $14 \%$ by weight; this was substantially less than that of the material under investigation in the present study. It has been shown that highly filled diacrylate resins have greater in vitro bond strengths than those with lower degrees of filler loading. ${ }^{2,16}$ In this respect the experimental light-cured material may be superior.

It was noted that $82 \%$ of the bond failures occurred in the first 6 months of the trial. There are three possible explanations for this. First, any deficiencies in the bond strength of any individual bracket/adhesive combination (caused by, for example, air inclusions, inadequate enamel surface etching, or poor moisture isolation) would become evident within this initial period of treatment. Second, this initial period of treatment is also a time of acclimatization for patients because they experience the restrictions concerning the type of food that can be masticated when wearing a bonded appliance. Unfortunately it is also a time of "experimentation" and consequently bond failures arise when restricted food is eaten. Third, the initial phase of treatment in many patients involves a period of overbite depression and, as a consequence, heavy occlusal forces may be applied to many of the bonded attachments, thereby resulting in bond failure.

The distribution of failure sites for the adhe- 
sive/bracket combinations is also similar when the failures are exarnined in an overall manner, in that there is a significantly greater number of posterior than anterior failures. This is likely to be caused by the increased difficulty of moisture isolation in the posterior segments of the dentition.

It could be suggested that the use of the light-cured material as described in this study is more time consuming than chemically cured material since it was necessary to cure each bracket individually. However, since maximum polymerization of the adhesive is achieved after exposure to the visible light source, it is therefore not necessary to delay arch wire placement, as is the case with chemical-cured materials in which arch wires cannot be placed until complete polymerization takes place.

Furthermore because of the command set of the light-cured material, there is also more time available to correctly position the bracket on the tooth surface; this is of great importance when placing preadjusted appliances. The handling properties of the visible light-cured materials are also superior to the chemically cured materials in that they are more viscous, which results in a reduction in the tendency for the brackets to slide on the enamel surface when they are first placed.

\section{CONCLUSIONS}

1. A visible light-cured adhesive material in combination with two types of bracket base was evaluated in a clinical trial and found to be a satisfactory alternative to a conventional chemically cured material. $\mathrm{Nei}$ ther material resulted in significantly different overall failure rates for any bracket base/adhesive combination.

2. When the site of bond failure was evaluated in an overall manner, there were significantly more posterior than anterior bond failures for all adhesive/base combinations.

We would like to acknowledge the assistance of Mrs. Lynne Lowe in typing the manuscript.

\section{REFERENCES}

1. Reynolds IR. A review of direct orthodontic bonding. $\mathrm{Br} \mathrm{J}$ Orthod 1975;2:171-8

2. Faust JB, Grego GN, Fan LF, Powers JM. Penetration coefficient, tensile strength, and bond strength of thirteen directbonding orthodontic cements. AM J ORTHOD 1978;73:512-25.

3. Lopez JT. Retentive shear strengths of various bonding attachment bases. AM J ORTHOD 1980;77:669-78.

4. Dickinson PT, Powers JM. Evaluation of fourteen direct-bonding orthodontic bases. AM J ORTHOD 1980;78:630-9.

5. Guzman R, Faust JB, Powers JM. Handling characteristics and bond strength of eight direct bonding orthodontic cements. I Clin Orthod 1980;14:631-6.

6. Gorelick L. Bonding metal brackets with a self-polymerizing sealant composite; a 12-month assessment. AM J ORTHOD 1977;71:542-53

7. Zachrisson BU. A posttreatment evaluation of direct bonding in orthodontics. AM J ORTHOD 1977;2:173-89.

8. Zachrisson BU, Brobakken BO. Clinical comparison of direct vs. indirect bonding with different bracket types and adhesives. AM J ORTHOD 1978:74:62-78.

9. Tavas A. Studies into orthodontic adhesives [PhD thesis]. Manchester University, 1980.

10. Read MJF. The bonding of orthodontic attachments using a visible light cured adhesive. Br J Orthod 1984;11:16-20.

11. Read MJF. Indirect bonding using a visible light cured adhesive. Br J Orthod 1987;14:137-41.

12. Tavas A. Watts DC. A visible light activated direct bonding material: an in vitro comparative study. Br J Orthod 1984; 11:33-7.

13. O'Brien KD, Watts DC, Read MJF. Residual debris and bond strength-Is there a relationship? AM J ORTHOD DENTOFAC ORTHOP 1988;94:222-30.

14. Lovius BBJ, Pender N, Hewage S, O'Dowling I, Tomkins A. A clinical trial of a light activated material over an 18 month period. Br J Orthod . 1987;14:11-20.

15. Fleiss JL. Statistical methods for rates and proportions. 2nd ed. New York: John Wiley \& Sons, 1982:173.

16. Buzzitta VAJ, Hallgren SE, Powers JM. Bond strength of orthodontic direct-bonding cement-bracket systems as studied in vitro. AM J ORTHOD 1982;81:87-92.

Reprint requests to:

Dr. K. D. O'Brien

Department of Orthodontics

University Dental Hospital of

Manchester and Turner Dental School

Higher Cambridge St.

Manchester M15 6FH, England 